\title{
Paediatric multisystem inflammatory syndrome in a ten-year old boy in a resource limited setting: a case for locally developed diagnostic criteria.
}

\author{
Animasahun $\mathrm{Ba}^{1 *}$, Lamina $\mathrm{Mo}^{2}$, Lawani $\mathrm{Fo}^{3}$, Akinola $\mathrm{Ao}^{4}$ \\ ${ }^{1}$ Department of Paediatrics and Child HealthLagos State University College of Medicine Ikeja. Lagos. Nigeria. \\ ${ }^{2}$ Consultant Paediatric Cardiologist Department of Paediatrics, Lagos State University Teaching Hospital Ikeja, Lagos. \\ ${ }^{3}$ Senior Registrar, Paediatric Cardiology, Department of Paediatrics, Lagos State University Teaching Hospital Ikeja, Lagos. \\ ${ }^{4}$ Senior Registrar, Paediatric Cardiology, Department of Paediatrics, Lagos State University Teaching Hospital Ikeja, Lagos. \\ *Corresponding Author: Animasahun Ba, Department of Paediatrics and Child HealthLagos State University College of Medicine Ikeja. \\ Lagos. Nigeria.
}

Received Date: February 25, 2021; Accepted Date: March 05, 2021 ; Published Date: March $10,2021$.

Citation: Animasahun Ba, Lamina Mo, Lawani Fo, Akinola Ao. (2021) Paediatric multisystem inflammatory syndrome in a ten-year old boy in a resource limited setting: a case for locally developed diagnostic criteria. Journal of Cardiology Research and Reports, 3(1): Doi: 10.31579/2692$9759 / 016$

Copyright: (c) 2021, Animasahun Ba. This is an open-access article distributed under the terms of the Creative Commons Attribution License, which permits unrestricted use, distribution, and reproduction in any medium, provided the original author and source are credited.

\begin{abstract}
Paediatric multisystem inflammatory syndrome (PMIS) occurs two to four weeks following exposure to the severe acute respiratory syndrome coronavirus 2 (SARS-CoV-2), the virus responsible for coronavirus 2019 (COVID-19). It is characterized by the presence of persistent fever, inflammation and organ dysfunction.

We present AE a 10-year old Nigerian boy who presented to the children emergency room with a history of continuous and high grade fever with back and multiple joint pains. While on admission he developed cough, difficulty with breathing, bleeding from the gums and altered consciousness which progressed to loss of consciousness.

Complete blood count showed neutrophilia of $82.9 \%$, lymphopenia of $9.8 \%$, thrombocytopenia of 142,000 cells $/ \mathrm{mm} 3$, elevated erythrocyte sedimentation rate of $75 \mathrm{~mm} / \mathrm{hr}$, chest radiograph findings of ground glass appearance and consolidation in all lung lobes bilaterally with a positive reverse transcriptase-polymerase chain reaction test for COVID-19 which met the Centre for Disease Control criteria for the diagnosis of pediatric multisystem inflammatory syndrome.

The patient was treated with intranasal oxygen, transfused with fresh whole blood, analgesics and antifailure drugs. His condition however deteriorated while on admission and died on the seven days of admission.
\end{abstract}

Keywords: pmis- pediatric multisystem inflammatory syndrome; covid-19- coronavirus 2019

\section{Introduction}

Since the onset of the COVID-19 pandemic in 2019, notably fewer cases of COVID-19 infection have been reported in children globally in comparison to adults possibly because children generally experience milder disease [1,2]. An increasing number of cases of children with more severe illness and features overlapping with the established diagnostic criteria of Kawasaki disease have been described in literature [3-6]. This has been termed Paediatric multisystem inflammatory syndrome (PMIS). It may progress to critical illness requiring intensive care in patients who develop cardiac involvement and coronary artery aneurysms [4].

Paediatric multisystem inflammatory syndrome (PMIS) or multisystem inflammatory syndrome in children (MIS-C) is a systemic disease characterized by persistent fever, inflammation and organ dysfunction following exposure to severe acute respiratory syndrome coronavirus 2 (SARS-CoV-2), the virus responsible for coronavirus 2019 (COVID-19) infection [4]. The syndrome occurs two to four weeks after infection with SARS-CoV-2 [7]. It is a life-threatening condition that is uncommon, seen in 2 in 100,000 persons less than 21 years of age with a higher proportion of cases occurring in blacks, Hispanics or South Asian children [2].

A case definition for this disease was first published by the Royal College of Paediatrics and Child Health in April 2020, followed by the World Health Organization and then the Center for Disease Control and Prevention in the United States of America [7]. The lack of consensus in the published case definitions raises important questions which are demonstrated in the case described below. 


\section{Case Report}

A 10-year-old male who presented to the Children emergency room following referral from a secondary health facility with a 12 day history of fever and 7 day history of generalized body pains. Fever was continuous, high grade and temporarily relieved by oral analgesics (Paracetamol). Generalized body pains involved the lower back, knee and elbow joints. The pain was dull and aching in nature, continuous, with limitation of movement in involved joints, temporarily relieved by oral analgesics. No antecedent trauma or associated history of joint swelling. He presented at the referring hospital 3 days after onset of symptoms where he was managed with antibiotics and analgesics but persistent symptoms necessitated referral to Lagos State University Teaching Hospital (LASUTH) where he was admitted.

He developed a cough described as non-paroxysmal, non-barky and nonwhooping on the second day of admission. The cough was productive of whitish sputum and not associated with chest pain. There was associated difficulty with breathing which was noticed two days after the onset of cough. There was no history of orthopnoea, easy fatigueability, bilateral leg swelling or fainting episodes. He also developed bleeding from the gums while on admission. It was not preceded by trauma, had three episodes of bleeding on the day of onset, about 1-2mls per episode, bleeding aborts spontaneously with no history of bleeding from any other part of the body. No history of skin eruptions, swelling of the hands or feet and swelling or redness of the tongue. No history of abdominal pain, diarrhea and vomiting. No history of convulsions, irritability and confusion.

There was no history of similar complaints in any other member of the family or exposure to persons with similar complaints. Ante-natal, birth and post-natal periods were not adversely eventful. He achieved motor (gross and fine) and speech milestones at the appropriate age. His past results in school showed good academic performance. He had received all vaccines according to the National Program on Immunization schedule. He was the second of five children in a monogamous setting.

On examination at presentation, he was acutely ill-looking, febrile with a temperature of $39.3^{\circ} \mathrm{C}$, pale, not in respiratory distress, acyanosed in room air, with no significant lymphadenopathy and no pedal oedema. He was tachypnoiec with a respiratory rate of 42 cycles per minute with oxygen saturation of $92 \%$ in room air and equal vesicular breath sounds bilaterally. He was tachycardic with a pulse rate of 112 beats per minute which was regular, normal volume and synchronous with other peripheral pulses. He had normal blood pressure for age. The apex beat was located in the fifth intercostal space, mid-clavicular line with normal first and second heart sounds auscultated. Abdominal examination revealed nontender hepatomegaly with a palpable edge $10 \mathrm{~cm}$ below the right costal margin and non-tender splenomegaly with a palpable edge $5 \mathrm{~cm}$ below the left costal margin. Musculoskeletal system examination revealed tenderness and limitation of movement in the left and right elbow joints and left knee joint.

An initial diagnosis of Juvenile idiopathic arthritis was made. With onset of cough and difficulty with breathing while on admission along with evidence of respiratory distress in the setting of an on-going pandemic, a diagnosis of COVID-19 infection with a possibility of PMIS was entertained.

Full blood count showed anaemia with a packed cell volume of $21.2 \%$, white blood cell count of 8600 cells $/ \mathrm{mm} 3$ with neutrophilia of $82.9 \%$, lymphopenia of $9.8 \%$, and mild thrombocytopenia of 142,000 cells $/ \mathrm{mm}$ [3]. Erythrocyte sedimentation rate was elevated with a value of $75 \mathrm{~mm} / \mathrm{hr}$. Chest radiograph showed ground glass opacification and consolidation in all lung lobes bilaterally and a Reverse Transcriptase-Polymerase Chain Reaction (RT-PCR) test for COVID-19 was positive.

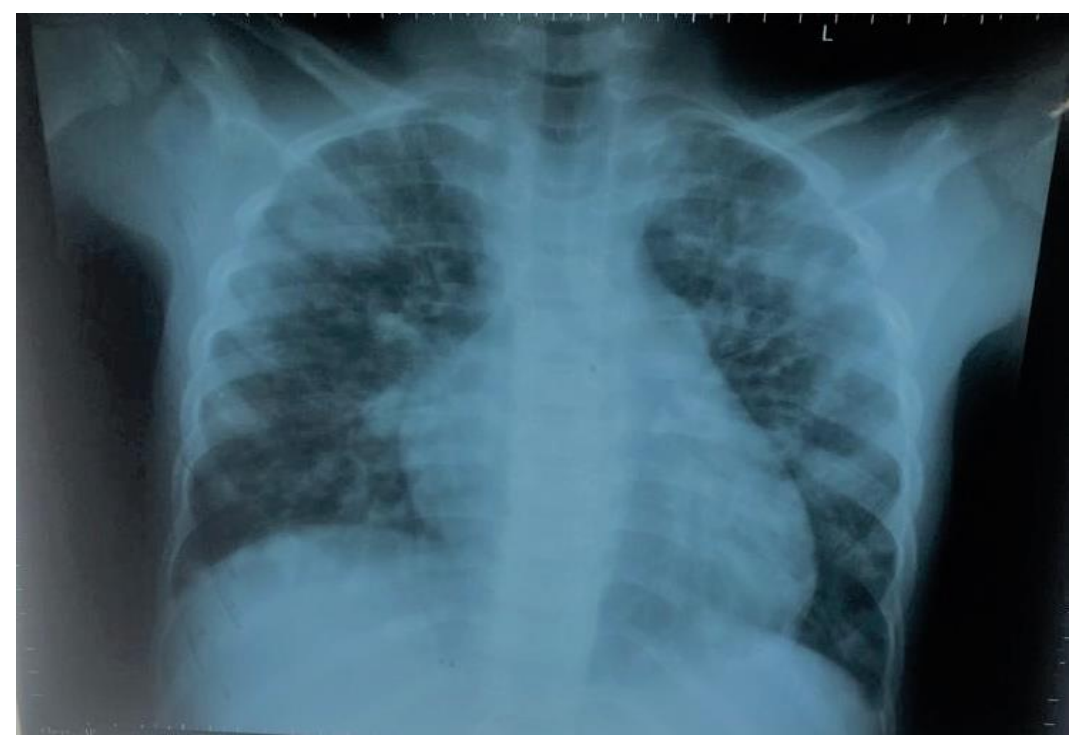

Figure 1: Chest Radiograph showing ground glass opacification and bilateral consolidation in all lung lobes bilaterally.

Other investigations requested included; C-reactive protein, clotting profile, cardiac inflammatory markers (Troponin $\mathrm{T}$ and $\mathrm{N}$-terminal proB-type natriuretic peptide) and echocardiography. However, these could not be done due to financial constraints.

He was commenced on intranasal oxygen, transfused with fresh whole blood and commenced on intravenous antibiotics. He developed neck stiffness and gradually declining level of consciousness seven days into admission with no obvious cranial nerve deficits, signs of meningeal irritation or elevated intracranial pressure. Clinical condition declined despite interventions until he developed cardiopulmonary arrest. All efforts at resuscitation proved abortive and he was certified dead after seven days on admission.

\section{Discussion}

The understanding of Paediatric Multisystem Inflammatory Syndrome (PMIS) is evolving as it is a relatively new disease entity, the first few cases being reported in April 2020. 
Since identification of the condition, attempts have been made at characterizing it with case definitions published by various organizations including the World Health Organization(WHO), Centre for Disease Control(CDC) and Royal College of Paediatrics and Child Health(RCPCH) [6]. Of importance is the lack of a consensus definition that incontrovertibly identifies or rules out a case being investigated as a possible case of PMIS [7]. All aforementioned criteria loosely agree on the presence of a fever and laboratory evidence of inflammation as necessary to make a diagnosis [6]. Beyond this, the differences in the criteria appear significant enough such that it is expected that they perform differently in identifying cases of PMIS [7]. This is highlighted in the case reported above as the patient only clearly meets the WHO and RCPCH criteria for PMIS, being 10 years old and having a fever lasting three weeks, heart failure, respiratory dysfunction, elevated ESR, neutrophilia, exclusion of microbial causes and a positive RT-PCR COVID-19 test.

Examining the three case definitions in more detail highlights the wide variability in the published criteria for making a diagnosis of PMIS. The WHO and CDC specify any fever lasting 3 or more days and a fever of $38^{\circ} \mathrm{C}$ or more lasting at least 24 hours respectively, while the $\mathrm{RCPCH}$ definition places no emphasis on duration of fever. While the RCPCH and WHO criteria make room for diagnosis in the presence of single organ dysfunction, the CDC criteria requires involvement of 2 or more systems. Only the WHO definition specifies the presence of mucocutaneous affectation, while the CDC definition alone includes presence of a "clinically severe illness requiring hospitalization". Perhaps most important is that the RCPCH definition does not require COVID-19 laboratory testing (PCR) to make a diagnosis of PMIS in contrast to WHO and CDC guidelines which require RT-PCR, Serology or antigen positivity or prior exposure to a COVID-19 positive individual [7].

These observations raise obvious questions: Are the clinical and laboratory features of PMIS dependent on geographical location and ethnicity? What are the features in children of African descent? Davies et al [5] published that up to half of 78 cases reported in a study in the UK were of Afro-Caribbean/Black descent while Feldstein et al [3] report that $25 \%$ of 186 cases reported in a US study were of similar descent. Twentynine (29) percent of the UK cohort presented with conjunctivitis in comparison to fifty-five (55) percent of the US cohort. Thirty-six percent (36) of the cases in the UK study had Coronary abnormalities, while only nine (9) percent of the cases reported in the US study had the same. What is the significance of these differences, especially when considered with respect to ethnic background of the reported cases? Should uniform criteria be applied across ethnicities? Whether or not the published case definitions are generalizable to children of African descent clearly remains to be seen and the need for further studies must not be ignored.

In resource-limited settings like in most of Nigeria, applying currently published case definitions may prove even more problematic as quite a number of the diagnostic modalities specified are not readily available. Such factors as echocardiography findings, elevated troponin/NTproBNP, CRP, procalcitonin, IL-6 etc. while they may be available in a limited number of centres in resource-limited settings are often out of the reach of the clinician due to prohibitive costs. The more readily available erythrocyte sedimentation rate (ESR) which was markedly elevated in the case reported above is only specified in the WHO and CDC case definitions but not the RCPCH definition, while neutrophilia as seen in the case reported only features in the RCPCH case definition.

The possibility of developing a more context-relevant case definition combining parameters of already published guidelines and emphasizing clinical features, backed by more readily available and ancillary laboratory parameters such as ESR, clotting profile, D-dimers, neutrophilia, lymphopenia, lactate dehydrogenase, hypoalbuminaemia with evidence of prior or present COVID-19 infection or exposure may prove to be a more pragmatic approach to identifying and ultimately managing PMIS in a resource-limited setting. In the US cohort involving 186 cases lymphopenia, hypoalbuminaemia, elevated ESR and INR were present in $\geq 75 \%$, while neutrophilia and elevated D-dimer were observed in about $70 \%$ [3]. It is also important to note that cardiac affectation features significantly in all three published case definitions, suggesting that a more encompassing case definition must delineate clearly features of cardiac involvement to make a diagnosis.

\section{Conclusion}

Paediatric Multisystem Inflammatory Syndrome is an evolving clinical entity of global importance that does not spare children in resourcelimited settings like Nigeria as reported here. More attention should be given to development of context-relevant criteria for diagnosis and management particularly in resource limited settings in order to improve outcomes.

\section{References}

1. Castagnoli R, Votto M, Licari A, Brambilla I, Bruno R, Perlini S, et al. (2020) Severe Acute respiratory syndrome coronavirus 2 (SARS-CoV-2) infection in children and adolescents: A systematic review. JAMA Pediatrics.

2. Lu X, Zhang L, Du H, Zhang J, Li YY, Qu J, et al. (2020) SARS-CoV-2 Infection in Children. N Engl J Med.

3. Feldstein LR, Rose EB, Horwitz SM, Collins JP, Newhams MM, Son MBF, et al.( 2020) Multisystem Inflammatory Syndrome in U.S. Children and Adolescents. N Engl J Med. 383(4):334-46.

4. Pouletty M, Borocco C, Ouldali N, Caseris M, Basmaci R, Lachaume N, et al. (2020) Paediatric multisystem inflammatory syndrome temporally associated with SARS-CoV-2 mimicking Kawasaki disease (Kawa-COVID-19): A multicentre cohort. Ann Rheum Dis. 79(8):999-1006.

5. Davies P, Evans C, Kanthimathinathan HK, Lillie J, Brierley J, Waters G, et al. (2020) Intensive care admissions of children with paediatric inflammatory multisystem syndrome temporally associated with SARS-CoV-2 (PIMS-TS) in the UK: a multicentre observational study. Lancet Child Adolesc Heal. 2(20):1-9.

6. Whittaker E, Bamford A, Kenny J, Kaforou M, Jones CE, Shah P, et al. (2020) Clinical Characteristics of 58 Children With a Pediatric Inflammatory Multisystem Syndrome Temporally Associated With SARS-CoV-2. JAMA [Internet]. 324(3):259.

7. Levin M. (2020) Childhood Multisystem Inflammatory Syndrome - A New Challenge in the Pandemic. N Engl J Med [Internet].383(4):393-5. 